\title{
A Method and a Device for Diagnostics of the Functional State of Peripheral Vessels of the Upper Limbs
}

\author{
A. I. Zherebtsova*, E. A. Zherebtsov, A. V. Dunaev, K. V. Podmasteryev, A. V. Koskin, \\ and $\mathrm{O}$. V. Pilipenko
}

\begin{abstract}
The article suggests a method and a device for diagnostics of the functional state of peripheral vessels of the upper limbs, which provide identification of angiospastic disorders with a lower probability of false-negative result, allowing thereby the quality of diagnostics to be improved. The suggested approach is based on combined application of laser Doppler flowmetry and contact thermometry during an occlusion test. The obtained results can be used in various fields of medicine for the development of multi-functional non-invasive diagnostic systems for the diagnosis and prevention of diseases associated with changes in the functional state of peripheral vessels.
\end{abstract}

\section{Introduction}

The market of biomedical equipment offers a variety of medical instruments for diagnostics of the functional state of peripheral vessels. However, the use of the instruments for microcirculation monitoring is restricted due to a lack of conformance with a number of requirements, including the requirement of diagnosis reliability. According to the results of expert assessment, the probability of false-negative results of instrumental diagnostics of the microvascular bed exceeds 0.3. Therefore, improvement of currently used techniques and development of new diagnostic methods and devices for reliable detection of peripheral vascular dysfunction becomes an important task.

Laser Doppler flowmetry (LDF) is a unique method for minimally invasive optical diagnostics that allows the blood flow intensity in the microcirculatory bed to be assessed. It can also be used to detect and study collective rhythmical microcirculation processes [1]. However, the lack of methodologies for rapid and reliable diagnosis of microcirculation disorders on the basis of LDF curves hinders large-scale introduction of LDF into clinical practice [2]. On the other hand, it is well known that peripheral circulation disorders lead to disorders in heat

Turgenev Orel State University, Ore

* To whom correspondence should be addressed. supply to the body surface. This allows the blood flow intensity and the efficiency of blood supply through the microcirculatory bed to be assessed by thermometric and thermographic methods $[4,5]$.

Combined use of LDF and thermometry allows complex diagnostic criteria to be formulated. This approach appears promising for improvement of methods and devices for diagnosis of peripheral circulation disorders [6]. Occlusion tests provide an additional opportunity for assessing the functional state of the microcirculatory bed. Occlusion tests produce artificial ischemia conditions in tissues of a limb. When the blood flow occlusion is removed, the test allows the process of recovery of vascular tonicity to be observed [1].

The goal of this work was to improve the quality of diagnostics of the functional state of peripheral vessels of the upper limbs. For this purpose, a method and a device for identification of angiospastic disorders with a lower probability of false-negative result were suggested. The approach to diagnostics used in this work is based on combined application of laser Doppler flowmetry and contact thermometry during an occlusion test.

\section{Theoretical Substantiation of Diagnostic Principle}

The skin temperature depends on a variety of factors [7]. Analysis of their mutual interaction reveals the presence of a closed feedback loop of regulation of the under- 
lying tissue temperature: biological tissue temperature, perfusion vs. temperature function and its parameters, tissue perfusion, tissue temperature. Depending on the feedback sign, this feedback loop can produce several stable states with a trigger mechanism of transition between them.

Reduction of temperature of vascularized biological tissue leads to microvascular spasm (low perfusion level). Conversely, an increase in temperature is accompanied by opening of precapillary sphincters and an increase in the number of open capillaries (high perfusion level). The threshold temperature of transition between these two states depends on the functional state of peripheral vessels. According to the approach suggested in this work, to achieve a pronounced angiospastic state caused by abnormal pathological decrease in perfusion due to exposure to low temperature, LDF and temperature signals are recorded in a limb submersed in water.

Mathematical models of the interrelationship between blood supply parameters and skin temperature were reviewed to select an optimal mathematical description of the thermal response of human hand to the occlusion test and to determine the diagnostic parameters [8]. It was found that the Pennes' bioheat transfer equation was optimal for accurate description of the problem of heat transfer in highly vascularized soft tissues. Taking into account changes in tissue perfusion during the test and assuming that the object of study is cylindrical in shape (a finger), a modified Pennes' equation can be written as follows:

$$
\begin{aligned}
\rho_{\mathrm{ts}} c_{\mathrm{ts}} & \frac{\partial T}{\partial t}+r^{2} / r_{\max }^{2} W_{\mathrm{bl}}^{\prime}(t, T) c_{\mathrm{bl}}\left[T-T_{\mathrm{art}}\right]= \\
= & k(r, \varphi)\left[\frac{1}{r} \frac{\partial}{\partial r}\left(r \frac{\partial T}{\partial r}\right)+\frac{1}{r^{2}} \frac{\partial^{2} T}{\partial \varphi^{2}}\right],
\end{aligned}
$$

where $\rho_{\mathrm{ts}}$ is the tissue density, $\mathrm{kg} / \mathrm{m}^{3} ; c_{\mathrm{ts}}$ is the tissue heat capacity, $\mathrm{J} /(\mathrm{kg} \cdot \mathrm{K}) ; c_{\mathrm{bl}}$ is the blood heat capacity, $\mathrm{J} /(\mathrm{kg} \cdot \mathrm{K})$; $W_{\mathrm{bl}}^{\prime}(t, T)$ is the tissue perfusion function during the test, which takes into account the occlusion periods, $\mathrm{kg} /\left(\mathrm{m}^{3} \cdot \mathrm{s}\right) ; T$ is the tissue temperature, $\mathrm{K} ; T_{\text {art }}$ is the arterial blood temperature, $\mathrm{K} ; k(r, \varphi)$ is the heat conduction factor, $\mathrm{W} /(\mathrm{m} \cdot \mathrm{K}) ; t$ is the time, $\mathrm{s} ; r$ is the radial coordinate in the cylindrical coordinate system, $\mathrm{m} ; \varphi$ is the angular coordinate in the cylindrical coordinate system, deg.

The function $W_{\mathrm{bl}}^{\prime}(t, T)$ takes into account the stages of the occlusion test (pre-occlusion, occlusion, postocclusion) (2):

$$
\begin{gathered}
W_{\mathrm{bl}}^{\prime}(t, T)=W_{\mathrm{bl}}(t, T) \times \\
\times\left[1-\theta\left(t-t_{\text {beg.occl }}\right)+\theta\left(t-t_{\text {end.occl }}\right)\right]+W_{0},
\end{gathered}
$$

where $t_{\text {beg.occl }}$ is the occlusion beginning time, $\mathrm{s}$; $t_{\text {end.occl }}$ is the occlusion end time, c; $\theta(t)$ is the Heaviside function ( $\theta$-function); $W_{0}$ is the minimal perfusion during occlusion (biological zero).

Nonlinear coupling between the tissue temperature and perfusion during the pre-occlusion and post-occlusion periods is described by the function $W_{\mathrm{bl}}(t, T)$ :

$$
W_{\mathrm{bl}}(t, T)=\frac{W_{\max }(t) \cdot \exp \left[R \cdot\left(T-T_{\text {thres }}\right)\right]}{1+\exp \left[R \cdot\left(T-T_{\text {thres }}\right)\right]}+W_{\text {min }},
$$

where $R$ is the slope of perfusion increase with increasing temperature, $\mathrm{K}^{-1} ; W_{\min }$ is the perfusion at minimum temperature, $\mathrm{kg} /\left(\mathrm{m}^{3} \cdot \mathrm{s}\right) ; T_{\text {thres }}$ is the skin temperature at which the perfusion value is the average between $W_{\max }(t)$ and $W_{\min }, \mathrm{K}$.

A sharp increase in perfusion during the post-occlusion period can be explained by an increase in the arteriole lumen and opening of precapillary sphincters due to accumulation of metabolic vasodilators. Vasoactive substances are gradually washed out of the vascular bed, so that the perfusion drops to its initial level. The maximum perfusion $W_{\max }(t)$ changes with time as follows:

$$
W_{\text {max }}(t)=W_{\text {st }}+W_{\text {peak }} \cdot \exp \left(-\left|t-t_{\text {end.occl }}\right| / \tau\right),
$$

where $W_{\text {st }}$ is the maximum perfusion in the stationary mode before the occlusion test, $\mathrm{kg} /\left(\mathrm{m}^{3} \cdot \mathrm{s}\right) ; W_{\text {peak }}$ is the maximum perfusion immediately after removal of the occlusion, $\mathrm{kg} /\left(\mathrm{m}^{3} \cdot \mathrm{s}\right) ; \tau$ is the time constant of the maximum perfusion decrease after removal of the occlusion, s.

It was assumed that the perfusion was directly proportional to the microcirculation index (MI, perfusion units) determined using the LDF technique.

The suggested mathematical model was verified using the perfusion parameters obtained in test measurements of the skin temperature and MI. It was found that the model curve was in relatively good agreement with the experimental curve of the skin temperature. Analysis showed that the suggested model could be used for theoretical research into the effect of the physiological parameters of the microvascular bed on the parameters of recorded thermograms, allowing thereby the choice of parameters for diagnosis of angiospastic disorders to be substantiated.

\section{Experimental Studies}

An experimental device was suggested for implementation of the diagnostic method described above. The 


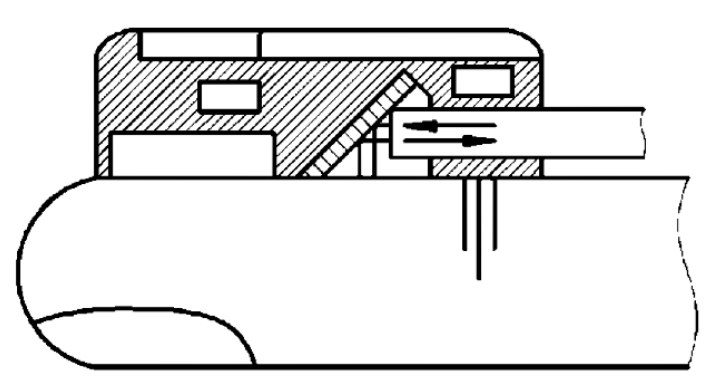

Fig. 1. Principle of operation of the adapter for fiber probes of LAKK devices.

LAKK-02 laser analyzer (OOO NPP Lazma, Moscow) was used to detect the microcirculation index. A twochannel contact thermometer with low inertia $(0.25 \mathrm{~s})$, low threshold of sensitivity $\left(0.05^{\circ} \mathrm{C}\right)$, and compact primary temperature transducers $(5.9 \times 2.1 \mathrm{~mm})$ was developed and manufactured. An adapter for light-guide probes of LAKK devices was developed to provide installation of the LDF probe over the skin surface. The principle of operation of the adapter based on $90^{\circ}$ light deflection is illustrated in Fig. 1.

Experimental studies of the functional state of peripheral circulation in fingers were performed in two groups: control and experimental. The control group consisted of 13 male and 14 female subjects (mean age, $23 \pm 5$ years) without diagnosed disorders of circulation, musculoskeletal system, or connective tissues (apparently healthy volunteers). The experimental group consisted of 5 male and 36 female subjects (mean age, $56 \pm 12$ years) undergoing inpatient treatment in the Orel Regional Clinical Hospital. The group included 26 patients with rheumatoid arthritis (stage II-III, activity degree II-III), 17 patients with joint arthrosis, 3 patients with systemic lupus erythematosus, one patient with ankylosing spondylitis, and one patient with gout. Patients with these

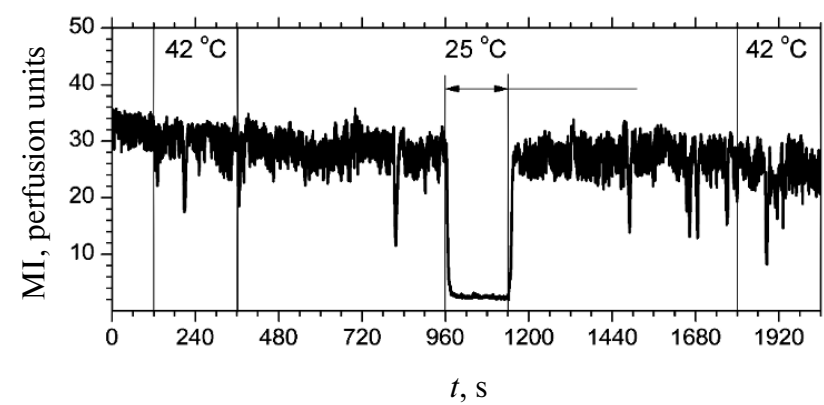

pathologies were included in the experimental group because of an early manifestation of microcirculation disorders that play a central role in the development of rheumatoid diseases [9]. During the test, LDF signals and temperature were measured simultaneously in the following stages [10]: 1) hand in air (basic test) - 2 min; 2) hand in water at $42^{\circ} \mathrm{C}$ (to provide identical initial conditions) $4 \mathrm{~min}$; 3) hand in water at $25^{\circ} \mathrm{C}-23 \mathrm{~min}$ (at this stage, 3min occlusion of the humeral artery is performed); 4) hand in water at $42^{\circ} \mathrm{C}$ (intensification of circulation) - 5$11 \mathrm{~min}$.

An example of simultaneous records of an LDF curve and skin temperature is shown in Fig. 2.

Potential diagnostic parameters were tested for sensitivity to the model parameters $\left(R, T_{\text {thres }}, \tau, W_{\text {peak }}\right)$ in order to substantiate selection of diagnostically valuable parameters. The parameters $\left(R, T_{\text {thres }}, \tau, W_{\text {peak }}\right)$ were selected because vascular bed pathologies usually affect their values. The temperature response index (TRI, arbitrary units) was found to be the most sensitive among the parameters under consideration:

$$
\mathrm{TRI}=\frac{\left(T_{\text {postoccl }}-T_{\text {in }}\right) /\left(T_{\text {occl }}-T_{\text {in }}\right)}{V},
$$

where $T_{\text {occl }}$ is the minimum temperature of biological tissue during the occlusion period, ${ }^{\circ} \mathrm{C} ; T_{\text {postoccl }}$ is the maximum temperature of biological tissue during the postocclusion period, ${ }^{\circ} \mathrm{C} ; T_{\text {in }}$ is the water temperature during the third stage of the test, ${ }^{\circ} \mathrm{C} ; V$ is the volume of the distal phalanx of the tested finger, $\mathrm{cm}^{3}$.

A combined diagnostic criterion was suggested for complex assessment of the state of the microcirculatory bed to minimize the diagnostic errors. The combined criterion includes both skin thermometry and LDF parameters.

The flow reserve index (FRI) is known to be especially useful for assessing the adaptation reserves of the

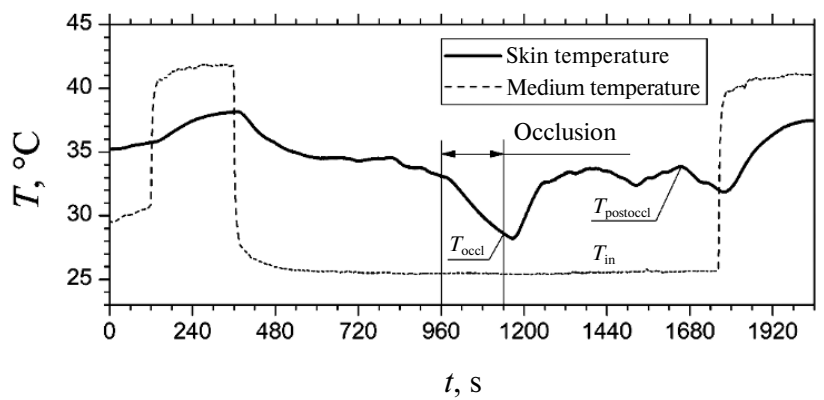

Fig. 2. Example of simultaneous experimental records of (a) LDF curve and (b) skin temperature (norm). 
microcirculation system by the LDF method using the occlusion test. The flow reserve index can be determined by the following equation [1]:

$$
\mathrm{FRI}=\left(\mathrm{MI}_{\max } / \mathrm{MI}_{\mathrm{in}}\right) \cdot 100 \%,
$$

where $\mathrm{MI}_{\max }$ is the mean microcirculation index during the first $60 \mathrm{~s}$ after removal of the occlusion (perfusion units); $\mathrm{MI}_{\text {in }}$ is the mean microcirculation index $60 \mathrm{~s}$ before the occlusion test.

It was shown experimentally that the parameters FRI and TRI meet the requirements for statistical independence and significance of difference in their values calculated for apparently healthy volunteers and patients with rheumatic diseases. The following decision rule was synthesized to identify patients with angiospastic disorders:

$\left\{\begin{array}{l}\text { FRI } \cdot 0.028+\text { TRI } \cdot 2.033-4.15>0, \text { norm; } \\ \text { FRI } \cdot 0.028+\text { TRI } 2.033-4.15 \leqslant 0, \text { angiospastic disorders. }\end{array}\right.$

The decision rule was verified using the leave-oneout technique. It allowed the probability of false negative result of diagnosis to be revealed at a level of 0.12 (considerably below the current level of error probability).

The discriminant curve was constructed so as to provide simultaneously a highly sensitive diagnostic criterion (0.88) and sufficiently high specificity (0.74). The sensitivity-to-specificity ratio can be changed by varying the coefficient of the equation describing the discriminant curve. The ROC curve for the suggested method of diagnosis is shown in Fig. 3. It indicates the quality of the suggested method of binary classification. The curve shows the sensitivity-to-specificity ratio at various values of the threshold between the domains of normal state of health and angiospastic disorders. The area under the curve is 0.88 , which is indicative of a high efficiency of the classifier.

Thus, this decision rule forms the basis of the suggested method for diagnosis of the functional state of peripheral vessels. It allows the flow reserve to be assessed using the LDF technique, while the responsiveness of deeply lying peripheral vessels can be evaluated using skin thermometry.

\section{Description of Diagnostic Device}

A device for diagnosis of the functional state of peripheral vessels of the upper limbs was suggested. The diagnostic procedure implemented in this device is based on combined application of LDF and contact thermometry during an occlusion test (Fig. 4).

The device operates by the following procedure. The operator enters the procedure parameters from the input unit and/or PC via the interface unit into the control unit. Signals generated by the control unit are transmitted to the LDF unit [11, 12], temperature unit, and compressor.

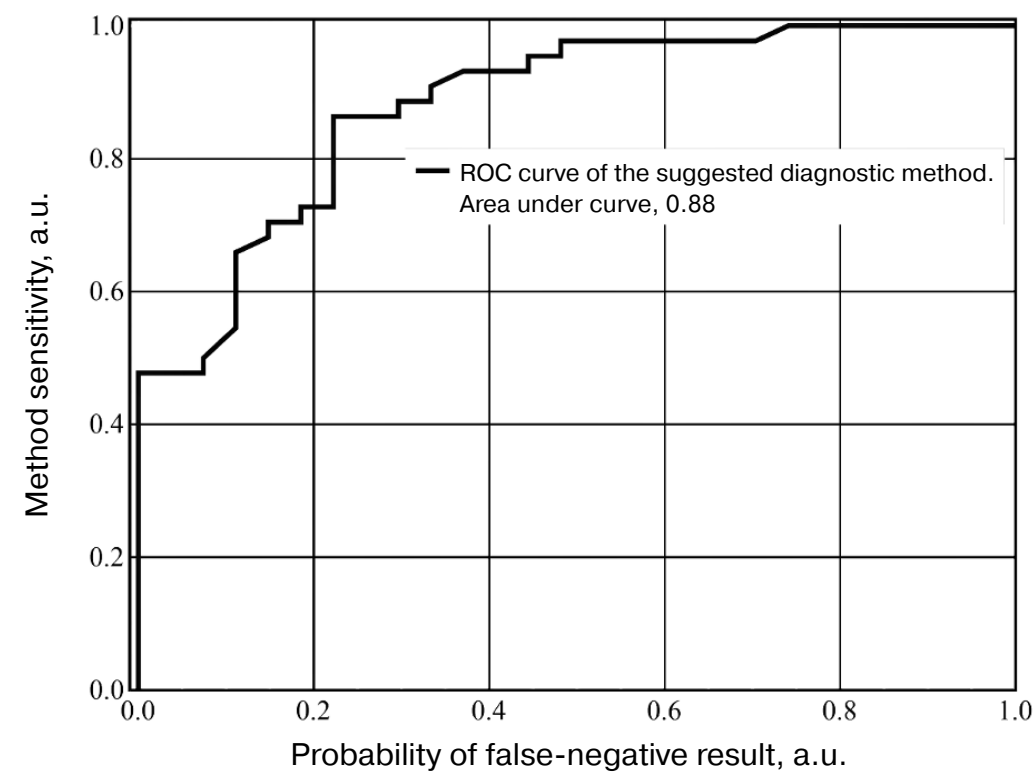

Fig. 3. ROC curve of the suggested diagnostic method 


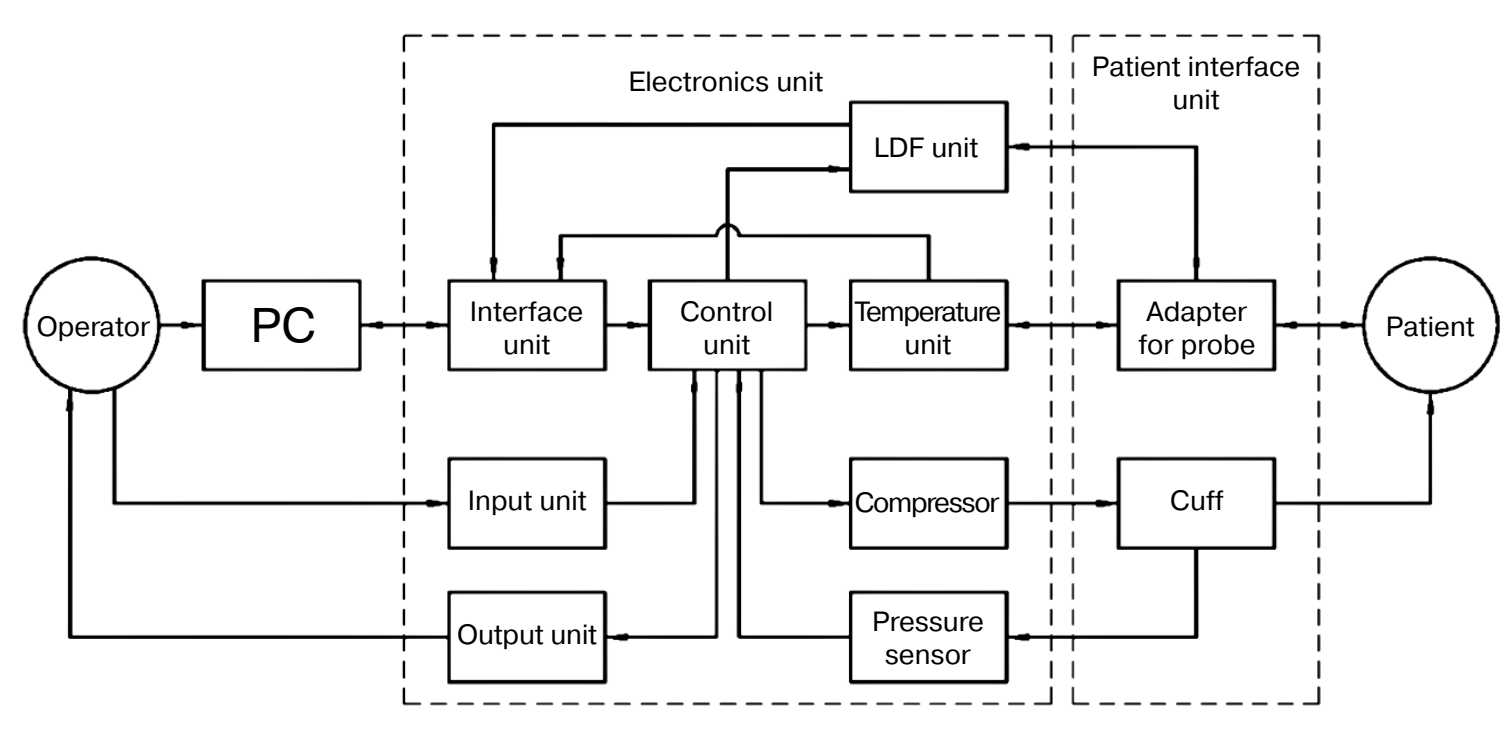

Fig. 4. Structural diagram of the device for diagnostics of the functional state of peripheral vessels of the upper limbs.

The light guide probe of the LDF device and primary temperature transducers are installed in the area of interest before the beginning of the procedure. An occlusion cuff is applied to the shoulder of the test subject. The operator initiates the procedure and monitors the stages of the test. The humeral artery is occluded by inflating the shoulder cuff with air using the compressor. The data on the air pressure in the cuff are transmitted from the pressure sensor to the control unit. Thus, the suggested device for diagnosis of the functional state of peripheral vessels allows simultaneous detection of the microcirculation index and the temperature by the LDF and contact thermometry techniques, respectively. The measurements can be performed during both basic and functional tests.

The diagnostic data are transmitted via the interface unit to the PC. Special software is used to calculate the FRI and TRI parameters. The decision rule is applied to make a conclusion about the functional state of peripheral vessels (presence or absence of angiospastic disorders). The structural diagram of one of the device modifications is presented in [13].

\section{Conclusion}

A method and a device for diagnostics of the functional state of peripheral vessels of fingers were developed. They provide identification of angiospastic disorders with a lower probability of false-negative result, allowing thereby the quality of diagnostics to be improved. The suggested approach is based on combined application of
LDF and skin thermometry during an occlusion test under thermostabilized conditions. The obtained results can be used in various fields of medicine for the development of multi-functional non-invasive diagnostic systems for the diagnosis and prevention of diseases associated with changes in the functional state of peripheral vessels.

This work was performed in the framework of the basic part of the State Program No. 310 of the Ministry of Education and Science of the Russian Federation for the Turgenev Orel State University and the Grant of the President of the Russian Federation for young scientists No. MK-7168.2016.8.

The authors are grateful to L. S. Khakhicheva, Head of the Department of Rheumatology, and V. F. Muradyan, Physician-in-chief, Orel Regional Clinical Hospital, for their help in conducting the experimental studies.

\section{REFERENCES}

1. Krupatkin, A. I. and Sidorov, V. V., Functional Diagnosis of the State of Microcirculatory Tissue Systems: Oscillations, Information, Nonlinearity (Manual for Physicians) [in Russian], LIBROKOM, Moscow (2013).

2. Dunaev, A. V., Novikova, I. N., Zherebtsova, A. I., Krupatkin, A. I., Sokolovskiy S. G., and Rafailov E. U., "Analysis of the physiological spread of parameters of microcirculatory tissue systems," Biotekhnosfera, 29, No. 5, 44-53 (2013).

3. Ley, O. and Deshpande, C.V. "Comparison of two mathematical models for the study of vascular reactivity," Comput. Biol. Med., 39, No. 7, 579-589 (2009). 
4. Usanov, D. A., Skripal', A. V., Protopopov, A. A., Sagaidachnyi, A. A., Rytik, A. P., and Miroshnichenko, E. V., "Assessment of the functional state of blood vessels by analysis of the temperature response to occlusion test," Saratov Nauch.-Med. Zh., 5, No. 4, 554-558 (2009).

5. Frick, P., Mizeva, I., and Podtaev, S., "Skin temperature variations as a tracer of microvessel tone," Biomed. Signal Process. Control, 21, 1-7 (2015).

6. Dunaev, A. V., Egorova, A. I., Zherebtsov, E. A., and Makarov, D. S., "A study of potentialities of thermal vision and noninvasive medical spectrophotometry for functional diagnosis," Fundament. Prikl. Probl. Tekhn. Tekhnol., 284, No. 6-2, 95-100 (2010).

7. Diller, K. R., Valvano, J. W., and Pearce, J. A., "Bioheat transfer," in: Kreith, F. (ed.) The CRC Handbook of Thermal Engineering, CRC Press, Boca Raton (2010), pp. 4-114-4-187.

8. Zherebtsova, A. I., "Analytical review of mathematical models of interrelationship between blood supply and skin temperature during an occlusion test," Fundament. Prikl. Probl. Tekhn. Tekhnol., 313, No. 5, 104-113 (2015).
9. Andrienko, A. V. and Bublikov, D. S., "Vegetative microcirculatory disorders in patients with rheumatoid arthritis," Fundament. Issl., No. 12, 163-166 (2013).

10. Zherebtsova, A. I., "A method for diagnosis of the functional state of peripheral vessels of fingers in patients with rheumatoid diseases," Fundament. Prikl. Probl. Tekhn. Tekhnol., 312, No. 4, 113-121 (2015).

11. Zherebtsov, E. A., Zherebtsova, A. I., Dunaev, A. V., and Podmaster'ev, K. V., "Method and device for metrological control of laser Doppler flowmetry devices," Biomed. Eng., 48, No. 4, 191-195 (2014).

12. Kozlov, I. O., Zherebtsov, E. A., Zherebtsova, A. I., and Dunaev, A. V., "The analysis of processing algorithms of laser Doppler signal in LabVIEW software," in: Proc. 2nd Int. Scientific Symposium Sense. Enable. SPITSE, St. Petersburg Electrotechnical University LETI, St. Petersburg, Russia (2015), pp. 225-227.

13. Dunaev, A. V., Zherebtsov, E. A., Egorova, A. I., and Rogatkin, D. A., "A device for diagnostics of the functional state of peripheral vessels," RF Patent No. 2503407; Application December 22, 2011; Published January 10, 2014; Bulletin No. 1. 